\title{
Surface plasmon resonance in the visible region in sensors based on tapered optical fibers
}

\author{
María-Cruz Navarrete ${ }^{\mathrm{a}}$, Natalia Díaz-Herrera ${ }^{\mathrm{b}}$, Agustín González-Cano $^{\mathrm{b}, *}$, Óscar Esteban $^{\mathrm{c}}$ \\ a Departamento de Óptica, Facultad de Ciencias Físicas, Universidad Complutense de Madrid, Ciudad Universitaria, 28040 Madrid, Spain \\ b Sección Departamental de Óptica, Escuela Universitaria de Óptica, Universidad Complutense de Madrid, Arcos de Jalón 118, 28037 Madrid, Spain \\ ${ }^{\mathrm{c}}$ Departamento de Electrónica, Universidad de Alcalá, Escuela Politécnica, 28871 Alcalá de Henares, Madrid, Spain
}

\section{A R T I C L E I N F O}

\section{Article history:}

Received 18 July 2013

Received in revised form 6 September 2013

Accepted 12 September 2013

Available online 25 September 2013

\section{Keywords:}

Tapered optical fibers

Surface plasmon resonance

Visible region

\begin{abstract}
A B S T R A C T
Doubly deposited uniform-waist tapered optical fibers (DLUWTs) have shown their versatility and good performance as basis for surface plasmon resonance (SPR) sensors for a variety of wavelength ranges. In this work we experimentally show how these devices can be employed to extend the technology of SPR fiber sensors to the visible region, down to about $530 \mathrm{~nm}$, with remarkable results in terms of sensitivity, plasmon definition and the availability of multiple plasmon resonances for each configuration. In this way, DLUWTs can be used to cover a range of more than $1000 \mathrm{~nm}$ for aqueous media only by changing the thickness of the deposits. Also, it is shown how these results can be used with the so-called absorption method to make selective the response of the sensors and a study is made on the influence of the taper waist in the performance of the devices. The number of SPR fiber sensors working in the visible region, of great interest in biological research, that have been depicted in the literature is very small, and the sensors that we present here notably improve their performance.
\end{abstract}

(C) 2013 Elsevier B.V. All rights reserved.

\section{Introduction}

Surface plasmon resonance (SPR)-based refractometry has become a standard technique in chemical and biological analysis in the last years, and many different devices have been presented in the literature [1-3]. Many of them are based on bulk configurations that use attenuated total reflection, but SPR optical fiber sensors offer a variety of advantages, including accessibility to difficult places, robustness, simplicity, small size, versatility, possibility of multiplexing, etc. For those reasons, the impulse to propose new concepts of SPR fiber sensors is strong, and the penetration of fiber technology in the fields of biosensing, chemical analysis and environmental control has considerably increased.

From the many options that can be chosen as a physical basis for SPR fiber sensors, doubly deposited uniform-waist tapered fibers (DLUWTs) have already shown their very good possibilities. It has been proven in the literature that with the very same concept, extremely simple, and only by changing the thickness of the deposited layers we can move their response to different wavelengths. In this way, the IR region has been covered from $800 \mathrm{~nm}$ to the $\mathrm{C}$-band around $1.5 \mu \mathrm{m}$ with remarkable results in terms of plasmon resolution and sensitivity [4-9], always adapting the response

\footnotetext{
* Corresponding author. Tel.: +34 913946903; fax: +34 913946885.

E-mail address: agus@fis.ucm.es (A. González-Cano).
}

of the systems to the refractive index range of aqueous media, the most interesting one in terms of biological applications. DLUWTs have, in this way, established themselves as one of the best design options for SPR fiber sensors.

The number of SPR fiber sensors working in the visible region for aqueous media is very small [10]. Some of them are based on plastic fibers and their performance is not very good [11]. The need of extending fiber technology into the visible region is self-evident, not only because the advantages that it can offer in terms of availability of sources, detectors and other components, but also because in the visible region we can fully take advantage of the so-called "absorption method" for SPR sensors, theoretically depicted and experimentally justified by the authors $[12,13]$, where selectivity can be achieved without the need of any added recognizing agents by tuning the plasmon resonances to the absorption wavelength of the analytes. Many interesting substances can be identified by their absorptions in the visible region, so we need devices that can provide tunable, well defined plasmon resonances for wavelengths of the visible region.

What we present in this work is a natural extension of DLUWT devices to this visible region. We have adapted the design of the transducers to move their response to wavelengths between 500 and $700 \mathrm{~nm}$, essentially by reducing the thickness of the dielectric layer (titanium dioxide in this case). We experimentally show in a very clear way how these plasmon resonances can be produced for outer refractive indices in the range of aqueous media 
and we obtain very well defined dips in the transmittance curves, that can be easily tracked to achieve a remarkable sensitivity for this part of the spectrum. Also, we count with multiple plasmon resonances, which is a peculiar characteristic of asymmetrically deposited DLUWTs, so we can use these varied dips for multiple measurements or to tune them to different components if we use the absorption method.

The fact that DLUWTs are based on single-mode fibers permit to improve evanescent field coupling, and we present here also an analysis of the dependence of the response of the devices with the diameter of the waist of the tapered region, showing a notable improvement in the performance when this diameter is reduced. As far as we know it is the first time that a SPR-based fiber sensor using single-mode fibers with resonances in the visible region for the refractive index of aqueous media is presented.

Also, this work permits us to "close the circle" by showing how the very same concept of DLUWTs, very simple in its essence and quite easy to implement, can be used to provide sensors that can work from about 500 to about $1500 \mathrm{~nm}$ (a range of about $1000 \mathrm{~nm}$ ) only by changing the configuration of the layers, and in our case only by changing their thickness, since the used materials are the same (titanium dioxide and aluminum). In all cases the performance of the produced sensors, as has been shown in different papers, is very good, in terms of sensitivity or plasmon resolution.

\section{Transducers fabrication and experimental setup}

The transducers have been produced with our usual technique. $[4,6]$ A traveling burner is employed to taper the fiber in a controlled way. We can control any parameter of the taper, and, most significantly in this paper, we can define the diameter of the waist, that has an important influence in the access to the evanescent field and therefore in the performance of the device. In the first measurements we have used a diameter of $30 \mu \mathrm{m}$, but, as we discuss in the following section, we have varied it down to $26 \mu \mathrm{m}$ with very good results. We have used a Newport FSA single-mode fiber.

Typically, we have produced taper lengths of $30 \mathrm{~mm}$ and waist lengths of 5-6 mm. These values are the usual with our DLUWTs. The profiles of the transition region have been chosen to provide smooth mode coupling into the waist, following the general philosophy of the use of uniform-waist (and not biconical) tapers. In that sense, the tapered fibers that we have used are essentially the same that we have used in the past to excite plasmons for other wavelength regions, and the variation has been introduced in the thickness of the deposited layers. For further and more detailed information about the characteristics of tapers, the dynamics of the coupling of light in the transition regions and the influence of the extension of the length of the deposited area, some of our references in the literature can be considered. [4-9] In this case our deposits completely cover the tapered region.

To define the thickness of the layers required to obtain those resonances we have run the simulation programs developed by us. In this case the thickness of the aluminum layer has been maintained in the value of $8 \mathrm{~nm}$, and the thickness of the dielectric layer, for which we have chosen our most common option, $\mathrm{TiO}_{2}$ (we have successfully tested InN in the past [14] but in this case this cannot be used because it is not transparent in this wavelength region), must be around $25 \mathrm{~nm}$ to provide resonances in the desired range. A scheme of one of the transducers used to provide the measures here presented is shown in Fig. 1.

If we compare these values with those used, for instance, to produce plasmons in the C-band, [8] we can see that both layers are much thinner in this case, which is, of course, logical, but it is also significant from the point of view of the fabrication. The layers have been deposited in a vacuum chamber with the e-beam technique

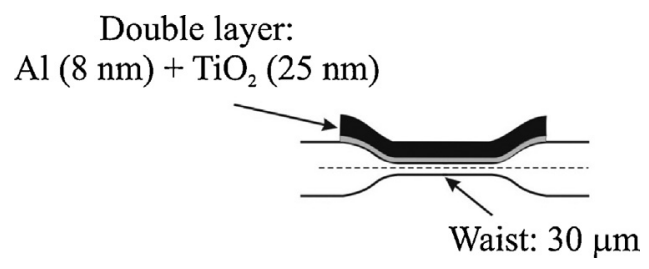

Fig. 1. Scheme of one of the transducers employed to produce plasmon resonances in the visible region.

and the fact that the total thickness is comparatively small makes more difficult the reproducibility, because any deviation in the deposition implies an increased relative error. This must be taken into account, because if we have a real thickness different from that desired (which is the one selected according to the simulations) the plasmon will subsequently move. However, as it can be seen in the experimental result we show, we have achieved to obtain resonances in the visible, going as deep as $530 \mathrm{~nm}$, when the usual range with these devices has always been around $800 \mathrm{~nm}$ for the refractive indices of the aqueous media.

The characterization procedure for the devices has also been repeatedly used in the past. $[4,6,8]$ It is basically a measure of the spectral transmittance of the transducer, when immersed in a mixture of water and ethylene glycol, whose refractive index can be varied in a controlled way by manipulating the concentration of ethylene glycol. The light source is a halogen lamp and a spectrometer is used as a measuring device. A polarizer is used to improve the response of the devices, although we have proved that the influence of the polarization in asymmetrically-deposited DLUWTs is quite small. A scheme of the characterization setup is shown in Fig. 2.

When the refractive index of the mixture of water and ethylene glycol changes, the position of the minima of the spectral transmittance curve is shifted. This displacement is the measuring parameter in this technique. The dips are associated to plasmon resonances, which are extremely sensitive to the variation of the refractive index of the surrounding medium. The slope of the (usually quite linear) curve that relates the position of these dips (the resonance wavelengths) to the refractive index is usually taken as an estimation of the sensitivity of the device.

\section{Experimental results}

A first and most important result of this work is the experimental evidence of the possibility of exciting plasmons in the visible region, near $500 \mathrm{~nm}$, with devices like those presented, based on singlemode tapered glass fibers (Fig. 3). This is a novelty in itself and, as we have said before, it is very interesting from the point of view of future applications in chemical and biological sensors. We have proven the feasibility of producing SPR fiber sensors in that region with DLUWTs. We can see that dips are well defined, quite narrow and deep, with transmittances of the order of 50-60\% (these have been improved in other devices, as commented below). The sensitivity for these transducers is of the order of $2000 \mathrm{~nm} / \mathrm{RIU}$, quite high, comparable to that obtained with sensors based on DLUWTs and of course much better than that reported for SPR fiber sensors working in the visible region. This sensitivity is estimated with the slope of the curve that relates the wavelength of the main resonance with the value of the refractive index of the surrounding medium, shown in Fig. 4.

As we have said before, we decided to investigate the influence of the waist diameter in the performance of the sensors. In a general way we can say that the narrower the waist the better the access to the evanescent field and the higher the response of the devices. So we tested two other diameters, namely, 28 and $26 \mu \mathrm{m}$. In Fig. 5 we show the results obtained. We must note that in principle we 


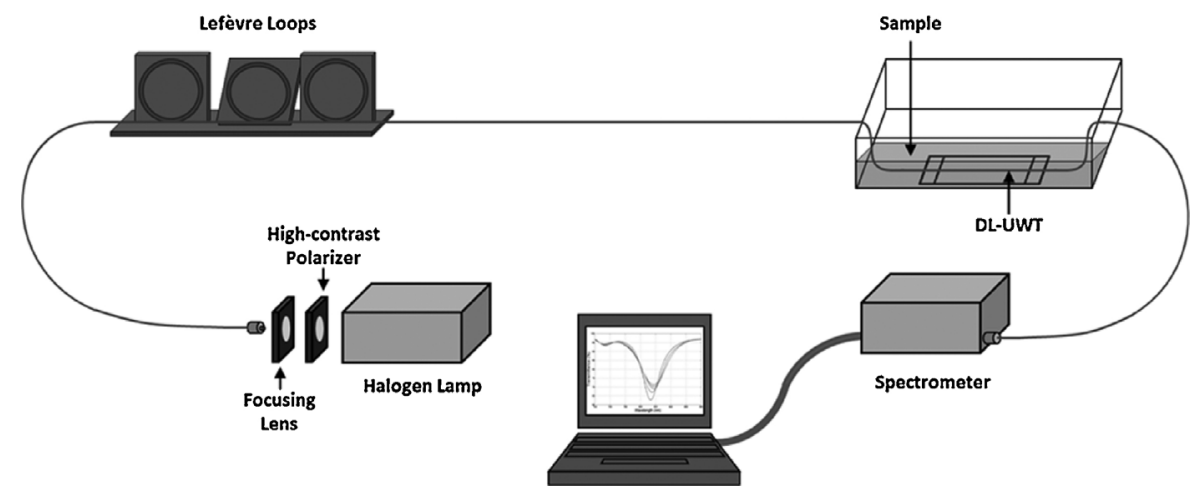

Fig. 2. Scheme of the experimental setup for the characterization of the transducers.

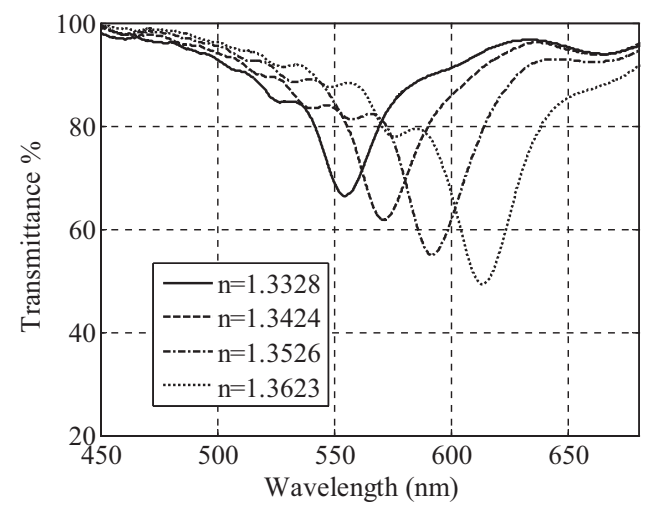

Fig. 3. Spectral transmittance of a tranducer with $8 \mathrm{~nm}$ of aluminum and $25 \mathrm{~nm}$ of titanium dioxide. The curves show the displacement of the plasmon dip with the variation of outer refractive index, always within the visible region.

have produced the sensors introducing the same initial parameters that in the other cases in the deposition chamber, and therefore we should expect resonances in the same values of wavelength. However, as we have said, the technical tolerances of the deposition process and the additional difficulties associated to the geometrical form of the tapers introduce deviations in the real obtained thicknesses that are more significant than in other larger wavelength regions. At the same time, it is always difficult to really establish the effective refractive index of layers so thin and therefore the algorithms (mostly empirical and highly dependent on the particular machine used) to determine the parameters to achieve a specific layer do not work properly. Because of that we estimate the real thickness of the layers of the sensors shown in Fig. 5 to be of the

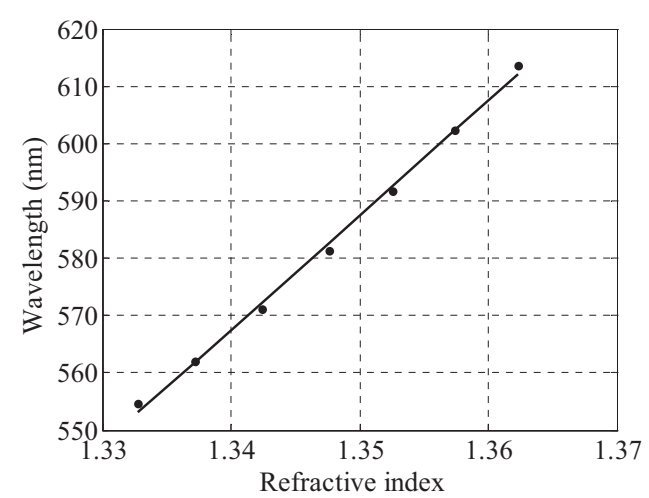

Fig. 4. Change in the resonant wavelength with the outer refractive index for the device of Fig. 1 whose spectral transmittance is depicted in Fig. 3. The slope of the curve permits to estimate the sensitivity of the device, $1998 \mathrm{~nm} / \mathrm{RIU}$. order of $15 \%$ higher than those intended and this is the reason that the resonances have been displaced to larger wavelengths. It is true (as we have said before) that this could be a problem if we try to exactly tune the response of the sensors to a particular wavelength, but it is a matter of reproducibility and repeatability very difficult to control in a physico-chemical process as deposition in a vacuum chamber. In a worst-case scenario this can imply that some of the sensors are not usable and this may require a more in-deep future treatment of the more subtle details of the deposition process, but, as our main goal was to prove that obtaining resonances in the visible, the fact that we have easily produced sensors able to guarantee that is enough for our purposes. Also, in terms of the behavior of the devices as SPR refractometres this, in fact, is no problem at all (assumed that we have a wide-range illumination spectrum, which is usually the case), because all we need is a relative measure, that of the displacement, and it is not relevant the absolute position of the particular resonances.

So, in this case the resonances are nearer to $700 \mathrm{~nm}$, but, as it can be seen, the fact that asymmetric DLUWTs provide multiple plasmon dips (a feature that is particular of that sensor type), we have resonances in a wide range within the visible region. As expected, the thinner the waist the better defined (deeper and narrower) the resonances, so it is advantageous to opt for a waist of the order of $26 \mu \mathrm{m}$, which can provide even a transmittance of the order of zero, a most remarkable fact, if we compare these results with those of Fig. 3 or with the ones of this Figure corresponding to a waist of $30 \mu \mathrm{m}$. It is quite rare to obtain transmittances as low (or plasmons as deep) as those. Also, the definition of all the other secondary resonances (up to the fourth one) is extremely good, so they can be perfectly used simultaneously or alternatively with good values of sensitivity (Fig. 6).

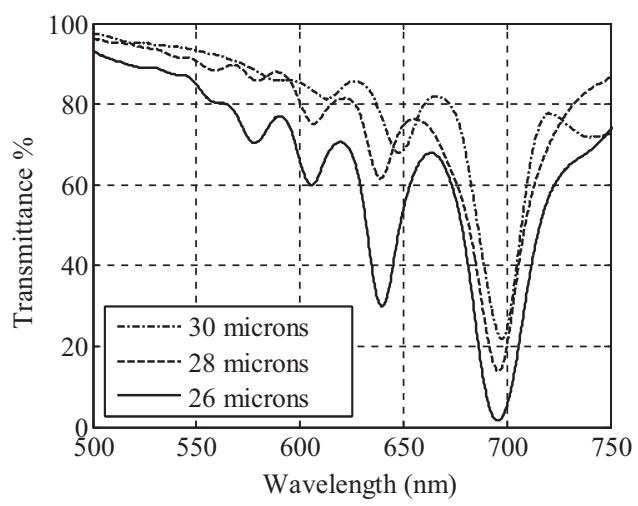

Fig. 5. Comparison of the performance of the devices with the variation of the waist diameter. Note the change in the depth of the dips, reaching almost a zero transmittance. Also, multiple plasmon resonances (up to the fourth one) are clearly marked. 
Table 1

Summary of the properties of SPR fiber sensors based on DLUWTs (representative values).

\begin{tabular}{|c|c|c|c|c|}
\hline Wavelength range & Thickness of $\mathrm{Al}(\mathrm{nm})$ & Thickness of $\mathrm{TiO}_{2}(\mathrm{~nm})$ & Sensitivity (nm/RIU) & Ref. \\
\hline $550-700 \mathrm{~nm}$ & 8 & 25 & $\sim 2000$ & This paper \\
\hline $750-850 \mathrm{~nm}$ & 8 & 60 & $\sim 4000$ & [7] \\
\hline C-Band, $\sim 1.5 \mu \mathrm{m}$ & 19 & 99 & $\sim 5000$ & [8] \\
\hline
\end{tabular}

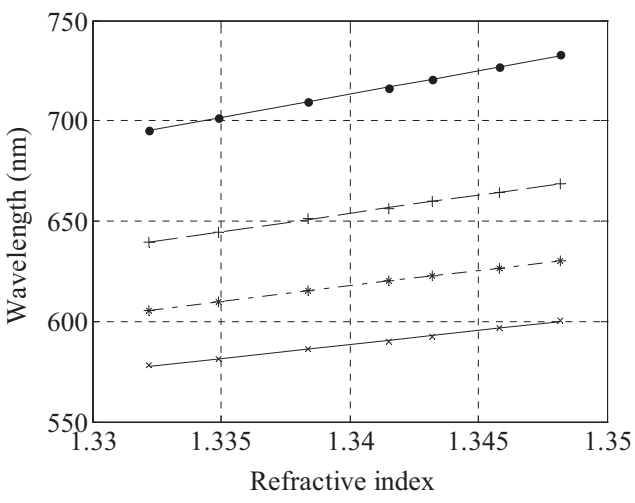

Fig. 6. Comparison of the displacement curves and estimation of the sensitivities for the four resonances of the device with $26 \mu \mathrm{m}$ of diameter waist. The slopes of the curves (and the corresponding sensitivity estimations) are (from the 1st dip, up, to the 4th one, below) $2332.8,1832.5,1541.7$ and $1380.3 \mathrm{~nm} / \mathrm{RIU}$ respectively.

In fact, this is a very important result since, as we have said, the possibility of tuning resonances with absorption wavelengths of the surrounding medium is the basis of a method that turns conventional SPR sensors in selective ones without the need of the addition of recognizing agents. Here we have four different resonances that can be associated to different substances. Also, we can track the various dips to increase the information of the system in different ways. In particular, with only one sensor we can extend its operating range to about $200 \mathrm{~nm}$ wide. The fact that the sensitivity of the measures associated with these dips is always high (as shown in Fig. 6) is also important. It can be seen that for the principal resonance the sensitivity is better than that corresponding to the results of Fig. 3, but also in this case the wavelength corresponding to these resonances is a little higher. It must be taken into account that the scale in the $y$-axis of Fig. 6 is quite different from that of Fig. 3, because it must cover a wider wavelength range.

\section{Conclusions}

We have presented experimental evidence of the possibility of exciting plasmons of an unusual depth and definition in the visible region for refractive index of the surrounding medium similar to that of water. This has been possible with the use of doublydeposited uniform-waist tapers (DLUWTs) with the right thickness in the layers of aluminum and titanium dioxide. We have analyzed the influence of the diameter of the taper waist, showing how the performance of the transducers is greatly improved with a narrower waist. Also, we have shown how multiple plasmon resonances can be excited with a resolution so good that with a single sensor one can cover, choosing the corresponding dip, a very wide range within the visible region. These secondary dips can be used simultaneously or alternatively to the principal one in several arrangements, notably in those based in the so-called "absorption method" to associate the resonances to absorption wavelengths of the analytes. To our knowledge, this is the first time that a SPR fiber sensor based on single-mode fibers is demonstrated for this combination of wavelengths and refractive indices, and the performance of the devices is comparable to those working in the more usual regions of the near infrared.
DLUWTs have been used in the past by the authors to provide a most convenient alternative to other procedures of exciting plasmons in several spectral regions. The advantages of DLUWTs include smallness, robustness, cheap fabrication, extreme versatility and the possibility of integration in systems working in real, hostile environments. In Table 1 we summarize some of the results presented in the literature to show how DLUWTs, with easy-toimplement changes in the thickness of the layers can cover a spectral range of more than $1000 \mathrm{~nm}$. As it can be seen, roughly speaking, for longer wavelengths we need thicker deposits, and we achieve higher sensitivities. Of course, a different choice of materials is also possible, but the couple $\mathrm{Al}-\mathrm{TiO}_{2}$ has proven to work perfectly. With this work, somehow we close the circle in the demonstration of the convenience of the option of tapered fibers as transducers based on evanescent field coupling, and the possibility of extending fiber technology to SPR chemical, biological and environmental sensing.

\section{Acknowledgements}

This work has been partially supported by Spanish Ministry of Science research projects SPRINT (reference CTQ2009-10550), by the Community of Madrid project FACTOTEM II (reference S2009/ESP-1781) and by the European Social Fund and the European Fund for Regional Development.

\section{References}

[1] J. Homola, Surface plasmon resonance sensors for detection of chemical and biological species, Chem. Rev. 108 (2008) 462-493.

[2] X. Fan, I.M. White, S.I. Shopova, H. Zhu, J.D. Suter, Y. Sun, Sensitive optical biosensors for unlabeled targets: a review, Anal. Chim. Acta 620 (2008) 8-26.

[3] I. Abdulhalim, M. Zourob, A. Lakhtakia, Surface plasmon resonance for biosensing: a mini-review, Electromagnetics 28 (2008) 214-242.

[4] A. González-Cano, F.J. Bueno, Ó. Esteban, N. Díaz-Herrera, M.C. Navarrete, Multiple surface-plasmon resonance in uniform-waist tapered optical fibers with an asymmetric double layer deposition, Appl. Opt. 44 (2005) 519-526.

[5] Ó. Esteban, N. Díaz-Herrera, M.C. Navarrete, A. González-Cano, Surface plasmon resonance sensors based on uniform-waist tapered fibers in a reflective configuration, Appl. Opt. 45 (2006) 7294-7298.

[6] F.J. Bueno, Ó. Esteban, N. Díaz-Herrera, M.C. Navarrete, A. González-Cano, Sensing properties of asymmetric double-layer covered tapered fibers, Appl. Opt. 43 (2004) 1615-1620.

[7] M.C. Navarrete, N. Díaz-Herrera, A. González-Cano, Ó. Esteban, A polarizationindependent SPR fiber sensor, Plasmonics 5 (2010) 7-12.

[8] N. Díaz-Herrera, A. González-Cano, D. Viegas, J.L. Santos, M.C. Navarrete, Refractive index sensing of aqueous media based on plasmonic resonance in tapered optical fibres operating in the $1.5 \mathrm{~m}$ region, Sens. Actuators B 146 (2010) 195-198.

[9] N. Díaz-Herrera, A. González-Cano, D. Viegas, J.L. Santos, M.C. Navarrete, Ó, Esteban, Moving the wavelength detection range in Surface Plasmon Resonance sensors based on tapered optical fibers, Proc. SPIE 7653 (2010) 76531F.

[10] M. Iga, A. Seki, K. Watanabe, Hetero-core structured fiber optic surface plasmon resonance sensor with silver film, Sens. Actuators B 101 (2004) 368-372.

[11] O.N. Cennamo, D. Massarotti, L. Conte, L. Zeni, Performance comparison of two sensors based on surface plasmon resonance in a plastic optical fiber, Sensors 13 (2013) 721-735.

[12] Ó. Esteban, A. González-Cano, N. Díaz-Herrera, M.C. Navarrete, Absorption as a selective mechanism in surface plasmon resonance fiber optic sensors, Opt. Lett. 31 (21) (2006) 3089-3091.

[13] I. Leite, M.C. Navarrete, N. Díaz-Herrera, A. González-Cano, Ó, Esteban, selectivity of SPR fiber sensors in absorptive media: an experimental evaluation, Sens. Actuators B 160 (2011) 592-597.

[14] Ó. Estaban, F.B. Naranjo, N. Díaz-Herrera, S. Valdueza-Felip, M.C. Navarrete, A. González-Cano, High-sensitive SPR sensing with Indium Nitride as a dielectric overlay of optical fibers, Sens. Actuators B 158 (2011) 372-376. 


\section{Biographies}

María-Cruz Navarrete received her M.Sc. degree in Physics in 1986, and her Ph.D. degree of Physics in 1994 in the Universidad Complutense de Madrid (UCM, Spain). She joined Optics Department (UCM) in 1990 where she has carried out her research and teaching activities. She has been involved in fiber optic research and in several European and Spanish projects. During the last fifteen years she has been working in optical fiber sensors, mainly applied to environmental measurements. Nowadays she is an assistant professor in the Optics Department and a member of the Applied Optics Complutense research group.

Natalia Díaz-Herrera received the M.Sc. degree in physics from the Universidad Autonoma de Madrid, Madrid, Spain, in 2001 and the Ph.D. degree in applied optics from the Universidad Complutense de Madrid (UCM) in 2005. In 2006 she joined the Optics Department of the UCM as a lecturer, where she has carried out her research and teaching activities. She has been involved in several European and Spanish projects. Her research interests are optical fiber sensors, specifically for environmental applications.

Agustín González-Cano was born in Madrid (Spain) in 1964. He received his M.Sc. in Physics (1987) and Ph.D. in Advanced Optics (1994) in the Universidad Complutense de Madrid (UCM), where he has developed his research and teaching activities during the last 26 years. Currently he is Professor in the School of Optics and Optometry of the UCM, where his main teaching subjects are Ophthalmic Optics, and Geometrical Optics. His research interests are basically optical sensors, and specifically fiber-optic sensors for environmental applications. In the past he has also been working in image processing, fringe pattern analysis and photoelasticity. In recent times he is also interested in research on the history of Optics. He has published more than 40 papers and has been involved in many different research projects, including some European Union-funded ones. He is a member of the Spanish Optics Association (SEDOPTICA).

óscar Esteban received his M.Sc. degree in Physics and the Ph.D. degree in Advanced Optics from Complutense University, Madrid, Spain, in 1997 and 2001 respectively. Since 2001, he has been with the University of Alcala, Spain, where he is currently Associate Professor in the Electronics Department and Head of the Photonics Engineering Group (GRIFO). He is also a member of the Optical Society of America (OSA). His main research subjects are optical-fiber sensors, optical testing of materials, thin solid films and optoelectronics applications. 